\title{
S20. Alcoholism: biological and psychosocial factors
}

EFFECTS OF INTRODUCTION OF A NEW ALCOHOLIC BEVERAGE ON ALCOHOL ABUSE IN THE COMMUNITY T Helgason. H Ólafsdóttir. $G$ Ásmundsson

Department of Psychiatry, National University Hospital, 101 Reykjavík, Iceland.

In 1989 beer containing more than $2.25 \mathrm{vol}$. \% alcohol was introduced to the market in Iceland. This resulted in an immediate increase in the average per capita consumption which has now levelled off. The increased consumption was expected to increase the prevalence of alcohol abuse in the community. Further, the consumption of the abusers was expected to increase proportionately more than the average consumption.

Methods. In order to test these hypotheses repeated postal surveys were carried out among random samples of the population aged 20-69 years, 1) in 1988 before the introduction of strong beer, 2) six months after the introduction, and 3) after three years. Alcohol abusers are defined operationally as those reporting three or more of eight symptoms commonly found in clinical work among alcoholics. Consumption is estimated from the selfreported quantity and frequency of alcohol use.

Results. The average consumption of the abusers was almost three times that of the non-abusers before the legislation of strong beer. In the two later surveys the average consumption of the non-abusers remained unchanged in the first and was reduced by almost $20 \%$ in the second, while that of the abusers doubled first after introduction of beer and was $25 \%$ higher in the final than in the first survey.

Conclusions. The legislation permitting sale of beer with an alcohol content exceeding 2.25 vol. \% has after three years not resulted in an increase in the average per capita consumption in the adult population. It has not caused any change in the prevalence of alcohol abuse, but the abusers are drinking more than previously.
DETERMINATION OF THE ALCOHOLISM FREQUENTLY IN THE POPULATION USING BLOOD ALCOHOL LEVELS OF SUICIDE CASES M. Berglund, A. Frank, P. Löwenhielm

Department of Alcohol and Drug Diseases, Malmō General Hospital, S-214 01 Malmö and Department of Forensic Medicine, University of Lund, Sölvegatan 25, S-223 62 Lund, Sweden.

The frequency of liver cirthosis in a population has been the standard parameter used for indirect calculations of the alcoholism frequency. In the present study another altemative, blood alcohol level of suicide cases, is presented.

Malmō with a population of 230.000 inhabitants offers unique possibilities for such studies. A large part of the population has been patients at the Department of Alcohol Diseases, for example $11 \%$ of the male and $3 \%$ of the female inhabitants born 1941. All suicide cases were subjected to forensic autopsy including measurements of the blood alcohol level, BAL and systematic evaluation concerning abuse (alcohol, narcotics and drugs) based on the police reports but without knowledge of hospital treatment. During 1984-87 there were 291 male suicide cases (included undetermined cases) and 125 female cases. 103 male suicide cases $(35 \%)$ and 22 female suicide cases (18\%) had previously been patients at the Department of Alcohol Diseases. An additional 40 male cases and 14 female cases were evaluated as abuse cases at autopsy. $30 \%$ of the registered male patients and $36 \%$ of the registered female patients did not get an abuse registration at autopsy. These groups had similar BAL levels as the abuse groups indicating active abuse. Based on these rates approximately another 17 male cases and 5 female cases were abusers not registered as patients or evaluated as abusers at autopsy.

$73 \%$ among the male patient abusers, $63 \%$ among the other male abusers and $28 \%$ among the other males had a positive alcohol test. Corresponding figures for females were $77 \%, 50 \%$ and $17 \%$, respectively. 42 male patient abusers $(41 \%), 15$ other male abusers $(38 \%)$ and 7 other males $(5 \%)$ had BALs over $0.2 \%$. Corresponding figures for females were $5(22 \%), 4(29 \%)$ and $3(3 \%)$, respectively.

In conclusion the rate of suicide together with the proportion of BALs over $0.2 \%$ could be used as a crude estimate of the number of alcoholics in the population. 
Influence of comorbidity on psychotropic consumption in alcoholic outpatients

\author{
JM Chignon 1-5 P Martin 2 , C Dissoubray $\underline{3}$, E Souetre 4 J Ades 5 \\ ${ }_{1}^{1}$ DPIM, service de l'evaluation des soins, AP-HP, 3 avenue Victoria 75100 \\ Paris-RP France \\ ${ }^{2}$ AMC Research Group, Hopital Robert Debré, 75019 Paris France \\ 3 Laboratoires Bristol Myers 92057 Paris La Défense France \\ ${ }^{4}$ Benefit Clinical and Quality of Life 92600 Asnieres-France \\ 5 Psychiatry departement, Hopital Louis Mourier, 178 rue des Renouillers \\ 92701 Colombes Cedex France
}

Several recent studies suggest that comorbidity with other psychiatric disorde

In a cross-sectional study including outpatients who met DSM-III-R criteria for alcohol abuse or dependance, we wanted to evaluate the consumption of different psychotropic agents and the impact of comorbidity, namely with depressive and anxiety disorders, on therapeutic strategies.

We include 507 patients ( 343 males and 164 females). The mean age at the intake of the study was 43.2 (SD:9.6) years. The lifetime prevalence of Major Depressive Episode was $49.1 \%$. The frequence of lifetime Panic Disorder and Agoraphobia were higher in females (respectively: $25.0 \%$ vs $15.7 \%, p<05 ; 21.3 \%$ vs $13.7 \%, p<05$ ). We found a high prevalence of Social Phobia and Generalized Anxiety Disorder without difference between females and males (respectively: $25.0 \%$ vs $20.7 \%$, NS; $56.1 \%$ vs $48.7 \%$, NS) Among these 507 patients, $392(77.3 \%)$ received at least one psychotropic with a large majority receiving benzodiazepines $(73.2 \%)$ and antidepressants $(45.7 \%)$. The anxiolytics and antidepressants consumption were found higher in females respectively: $(81.6 \%$ vs $66.9 ; p<01)$ and $(61.7 \%$ vs $36.7 \% ; p<01)$. Even if the existence of a depressive episode and anxiety disorders were associated with a prescription of antidepressants $(p<001)$ and anxiolytics $(p<001), 51.3 \%$ among patients without $p s y c h i a t r i c$ comorbidity received anxiolytics and $20.5 \%$ received antidepressants.

The different patterns of therapeutic strategies in alcoholic patients will be presented and discussed with logistic regression models integrating socio-demographic and clinical parameters.

\section{EMOTIONAL AND SOMATIC SYMPTOMS AND HEALTH ATTITUDES IN PATIENTS WITH ALCOHOL DEPENDENCE}

J Shlik, A Jāry

Department of Psychiatry, University of Tartu, 31 Raja Street, Tartu, EE2400, Estonia

Clinical observations are suggesting that bodily complaints and health anxiety are quite common among patients with alcohol dependence (AP). The aim of this study was to estimate somatic preoccupation and hypochondriacal features as well as their relationships to the emotional and somatic symptoms in AP. Assessment was performed in 40 consecutive patients $(35$ male and 5 female, mean age 39 $\pm 8,25-55$ years) who fulfilled the DSM-III-R criteria for alcohol dependence and 25 matched control subjects (NP). The exclusion criteria were current withdrawal state, significant somatic comorbidity and marked cognitive impairment. The emotional symptoms during last month were assessed by anxiety (HA) and depression (HD) subscales of the Hospital Anxiety and Depression Scale (Zigmond and Snaith, 1983). Intensity (SI) and number (SN) of somatic symptons experienced during last month were measured by Somatic Symptoms Inventory (SSI). Subscales of the Illness Attitude Scale (Kelliner, 1987) were used for assessment of worry about health (WH), concern about pain (CP), health habits (HH), hypochondriacal beliefs (HB), thanatophobia (TH), disease phobia (DP), bodily preaccupation (BP), treatment experience (TE) and effect of symptoms (ES). In addition both groups filled out the Toronto Alexithymia Scale (TAS; Taylor, 1988). Statistical methods applied for nonparametric variables were Mann-Whitney test and Spearman's rank correlation.

Some of the results are shown in the table as mean $\pm \operatorname{SD}\left({ }^{1}-p<.005 ;^{2}-p<.05\right)$.

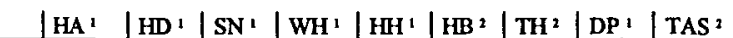

\begin{tabular}{l|r|r|l|l|l|l|l|l|l}
\hline $\mathrm{AP}$ & $10 \pm 4$ & $7 \pm 4$ & $9 \pm 8$ & $7 \pm 3$ & $5 \pm 3$ & $3 \pm 3$ & $4 \pm 4$ & $4 \pm 3$ & $70 \pm 12$ \\
$\mathrm{NP}$ & $5 \pm 4$ & $4 \pm 3$ & $2 \pm 2$ & $4 \pm 2$ & $8 \pm 3$ & $2 \pm 2$ & $2 \pm 2$ & $2 \pm 2$ & $63 \pm 10$
\end{tabular}

$H A$ and $H D$ scores correlated with $S I$ and $S N(r=0.5 ; p<05)$ and $H D$ also with DP, ES $(r=0.4, p<.005)$ and TAS $(r=0.4, p<.05)$ scores. SSI scores correlated with HB, TH $(r=0.4 ; p<.01)$, DP and ES $(r=0.5 ; p<.005)$

Results are demonstrating that patients with alcohol dependence are considerably affected by emotional and bodily symptoms as well as enduring hypochondriacal features and alexithymia. The somatoform-emotional interactions in the context of development and maintenance of alcohol dependence are clinically meaningful and worth of further research
MEMORIES OF PARENTAL REARING IN ALCOHOLICS DIVIDED ACCORDING TO THE FAMILY HISTORY

Radu Vrasti *) and Martin Eisemann **)

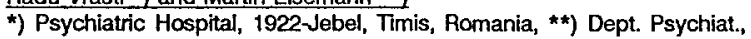
Umeå University, Sweden

It has been a well-established finding for two past decades that alcoholism runs in families. Thus, the incidence of alcoholism was fund to be statistically significant higher among offspring of alcoholics than offspring of non-alcoholics (Cotton, 1979). This observation imply a interaction of genetic and environmental factors. Between developmental factors involved in alcoholism the parental rearing practices were stressed by many authors (Beardslee et al. 1986; Brennan et al. 1991; Buchtholz et al. 1993; Campo \& Rohner, 1992; DeJong et al. 1991; Greenfield et al. 1993; Hill et al. 1992; Vrasti et al. 1990).

Subjects and methods:

84 male alcoholics who met DSM-III-R diagnostic criteria of alcohol abuse/dependence were divided according to the family history of alcoholism in the first degree relatives in two groups: FH + encompassing 40 alcohotics (mean age $38.5 \pm 8.0$ ) and FH-comprising 44 alcoholics (mean age $40.4 \pm 9.5$ ).

The EMBU questionnaire (Perris et al. 1980) was used to assess the memories of perceived parental rearing behaviour.

A control group from general population was also used.

Results and discussions:

As a whole, alcoholics reported parental rejection much more than controls, meaning more often corporal punishment, hostility and humiliation. The same results reported De Jong et al. (1991) using the same instrument, or Campo \& Rohner (1992) and Buchholz et al. (1993) with other methods. Surprising, no significantly differences on EMBU factors between alcoholics divided according to the family history were carried out.

The last finding might reflect the deleterious effect of having a parental rejection during childhood as an additional factor to a genetic predisposition.

SEROTONERGIC DYSFUNCTION IN ALCOHOLISM AND SENSORY EVOKED CORTICAI DIPOLE ACTIVITY Hegerl $U^{\mathrm{a}, b}$, Juckel $G^{\mathrm{a}, b}$, Lipperheide $K^{\mathrm{a}}$, Schmidt $\underline{L} G^{b}$. Rommel spacher $H^{b}$

Department of Psychiatry, Freie Universităt Berlin

a) Laboratory of Clinical Psychophysiology

b) Clinical Research Group on Neurobiological Mechanisms of Addiction, Eschenallee 3, 14050 Berlin, Germany

A large body of data suggests that alcoholics with antisocial, aggressive and impulsive behavior form a subgroup, in which a dysfunction of the brain serotonin system is of pathogenetic importance. Furthermore, it has been proposed that, in addition to antisocial behavior, early onset and a transmission from fathers to sons (type II alcoholism according to Cloninger 1991) are aspects characterising patients with a serotonergic dysfunction. A pronounced amplitude increase of responses of primary auditory cortices with increasing loudness of the stimuli, measured with auditory evoked dipole source activity, is supposed to indicate low serotonergic neurotransmission and vice versa. Evoked dipole activity to stimuli in 5 different intensities were recorded in 53 hospitalised patients one week after withdrawal. A significant stronger intensity dependence of the tangential dipole was found in the patients with antisocial tendencies ('legal problems', 'driving problems'. and abnormalities in social behavior in childhood and adolescence'). Age at onset and family history were not related to the intensity dependence of the evoked responses. The results suggest that alcohol dependent patients with a stronger intensity dependence form a subgroup with a serotonergic hypofunction and may be responders to a relapse prevention with serotonergic drugs. 
DOUBLE BLIND CONTROLLED STUDY VERSUS PLACEBO TO ASSESS THE EFFICACY AND TOLERANCE OF ACAMPROSATE IN A MULTICENTRE GERMAN STUDY ("PRAMA")

H. Sass, Psychiatrische Klinik, 52057 Aachen, Germany

In a randomised, double blind design 136 patients received acamprosate treatment and 136 received placebo. In addition to the usual recording of patients' history and demographic details, extensive assessments of psychological status and social environment were performed. According to the protocol, the main criterion of efficacy was the time to the first relapse. The cumulative duration of abstinence was also investigated. GGT, MCV, and CDT were measured as objective criteria. $134(49,38)$ patients completed the study after one year. The most sensitive indicator of efficacy proved to be cumulative duration of abstinence. The mean value in the acamprosate group was 178,5 days compared to 113,8 days in the placebo group. The difference between treatment groups is highly significant, with a p-value of 0.0001 . The differences were reflected in a similar way in the life-tables. A very marked difference between acamprosate and placebo treatment developed between 30 and 90 days after the start of the therapy. The absolute value of this difference increased only slightly on further treatment. The Log Rank test, which is the usual method of statistical comparison of life-table data, yielded a similar result $(p=0.0054)$. At the end of the study $42,8 \%$ of patients were abstinent in the acamprosate group and 20,78 in the placebo group. Further research concerning psychopathological, psychosocial and psychotherapeutical aspects, still being under evaluation, will be discussed. 